\title{
Olfaction and Colour Vision: What Can They Tell Us about Parkinson's Disease?
}

\author{
Irene Dall'Antonia, Karel Šonka, Petr Dušek \\ Department of Neurology and Centre of Clinical Neuroscience, \\ First Faculty of Medicine, Charles University and General University Hospital \\ in Prague, Prague, Czech Republic \\ Received April 24, 2018; Accepted October 5, 2018.
}

Key words: Hyposmia - Colour vision - Neurodegeneration - Synuclein

\begin{abstract}
Parkinson's disease is a neurodegenerative disorder with the pathological accumulation of alpha synuclein in the brain and peripheral nerve tissue. Early stages of synucleinopathies, often present clinically with rapid eye movement (REM) sleep disorder (RBD). Clinical markers that indicate early progression from RBD to manifest synucleinopathies include abnormal dopamine transporter (DAT) imaging, motor and non-motor symptoms. Despite the high diagnostic strength of DAT imaging and motor abnormalities, they are not the earliest biomarkers. Non-motor signs of neurodegeneration such as colour vision and olfaction abnormalities are detectable by clinical examination as early as 20 years before disease onset. Detailed analysis of olfactory and colour vision dysfunction can provide valuable information regarding brain pathologies, further specifying clinical phenotypes, and giving clues to underlying pathophysiological mechanisms in Parkinson's disease and related disorders.
\end{abstract}

This study was supported by the grant no. AZV 16-28914A of the Czech Ministry of Health of the Czech Republic.

Mailing Address: Irene Dall'Antonia, MD., Department of Neurology and Centre of Clinical Neuroscience, First Faculty of Medicine, Charles University and General University Hospital in Prague, Kateřinská 30,120 00 Prague 2, Czech Republic; Phone: +420 224965 009; e-mail: 21983@vfn.cz 


\section{Introduction}

Parkinson's disease (PD) belongs to the synucleinopathies, distinct neurodegenerative syndromes that are associated with progressive aggregation of alpha-synuclein in the nervous system. This group of disorders includes, additionally to PD, dementia with Lewy bodies (DLB) and multiple system atrophy (MSA) (Spillantini and Goedert, 2000). PD was first described by James Parkinson in 1817, however the histopathological abnormalities of PD were found almost 100 years later. In 1912, Friedrich Lewy discovered large eosinophilic neuronal kidney-shaped inclusions and thread-like structures now known as Lewy bodies and Lewy neurites respectively (Goedert et al., 2013).

Neurodegeneration in PD, DLB and MSA is linked to the process of accumulation of pathological alpha-synuclein aggregates, described by Friedrich Lewy in the brain, forming cytoplasmic inclusions in the central and peripheral nervous systems (Marques and Outeiro, 2012). Current research suggests that the spread of alpha-synuclein pathology in the nervous system may occur in a prion-like process (Bernis et al., 2015). This theory is supported by several animal and cell culture studies, as well as, neuropathological findings in transplanted fetal grafts (Halliday et al., 2011). The theory supports the notion that the synuclein pathological molecular template spreads inter-synaptically along neurons. On the other hand, different hypotheses about alpha-synuclein propagation have been postulated including neuroimmune activation, injury response, and aberrant proteostasis (Uchihara and Giasson, 2016).

Alpha-synuclein pathology is present years or decades before affected subjects fulfil clinical criteria for a particular synucleinopathy syndrome. This pathology is mirrored by mild clinical abnormalities such as isolated rapid eye movement (REM) sleep behaviour disorder (RBD), hyposmia, autonomic dysfunction including constipation and orthostasis, minor motor impairment manifested as speech changes and impaired dexterity and abnormalities on dopamine transporter (DAT) imaging (Postuma and Berg, 2016). Based on these symptoms, the prodromal stage of synucleinopathies was defined (Mahlknecht et al., 2015). From these abnormalities, RBD is a biomarker with by far the strongest association with synucleinopathies. It was discovered in 1986 by Schenck et al. and was thereafter classified as a parasomnia. A strong link between RBD and synucleinopathies was not unveiled until several decades later. In 1996, it was demonstrated that patients with RBD progressively convert to parkinsonism and this discovery opened a new period in synucleinopathy research (Schenck et al., 2013).

The initial clinical symptoms in prodromal PD and later in clinically established PD are in concordance with the pathological staging put forward by Braak et al. (2003). According to this theory, alpha-synuclein pathology initially affects the most vulnerable long unmyelinated nerve fibres of the autonomic nervous system and the anterior olfactory nucleus, later proceeding to the brain stem and olfactory bulb, and finally reaching the mesocortex, association and primary sensory-motor 
cortical areas (Braak et al., 2003). Olfaction dysfunction, constipation, RBD as well as a combination of other motor and non-motor symptoms form the diagnostic criteria for prodromal PD. Colour vision abnormalities are like olfactory dysfunction present several years before the onset of PD (Postuma et al., 2011). These markers can alert us to an early disease process, years before PD diagnosis, providing a unique opportunity to better understand the pathogenesis of synucleinopathies.

\section{Synucleinopathies: Clinical presentation and diagnostic criteria}

The main clinical symptom of synucleinopathies is parkinsonism, its responsiveness to levodopa and the presence of other symptoms helps in distinguishing the main three clinical syndromes.

The essential diagnostic criterion for PD is the presence of parkinsonism, which is defined as bradykinesia plus rigidity and/or tremor. In addition to these cardinal motor symptoms, diagnosis of clinically established PD requires absence of absolute exclusion criteria such as cerebellar dysfunction or vertical gaze palsy, absence of red flags such as rapid progression of gait impairment, no progression for five years, or early bulbar dysfunction, and presence of at least two supportive criteria. The latter are positive features that increase confidence of the PD diagnosis; olfactory loss is one of them (Postuma et al., 2015a).

Recently, diagnostic criteria for prodromal PD, understood as the disease stage before overt motor syndrome manifestation, has become available. These diagnostic criteria include non-motor features such as RBD, constipation and other symptoms of autonomic dysfunction, daytime sleepiness, depression, and abnormal DAT imaging (Berg et al., 2015).

The diagnosis of probable DLB requires progressive cognitive decline affecting predominantly attention, executive function, and visuoperceptual abilities along with at least two from the core features: profoundly fluctuating cognition, parkinsonism, RBD, and recurrent visual hallucinations. Supportive clinical features include sensitivity to antipsychotics, postural instability, repeated falls, syncope, autonomic dysfunction, hypersomnia, delusions, apathy, depression, anxiety, and hyposmia (McKeith et al., 2017).

MSA is a sporadic, progressive disorder with onset of symptoms after 30 years of age. The diagnosis of probable MSA requires the following abnormalities: autonomic dysfunction and a levodopa poorly responsive parkinsonism or a cerebellar syndrome (Gilman et al., 2008). Apart from the listed core criteria, there are also supportive criteria including severe dysarthria, dysphonia and dysphagia, postural abnormalities such as camptocormia or antecollis, inspiratory sighs and snoring. Contrary to PD and DLB, there are distinctive MRI signs, which can be used to support the diagnosis of MSA; these include atrophy of the putamen, middle cerebellar peduncle, and the pons along with $\mathrm{T} 2$ signal abnormalities including posterior putaminal hypointensity, middle cerebellar peduncle hyperintensity, 
and the hot cross bun sign in the pons (Brooks et al., 2009). Of note, hyposmia is uncommon in MSA; RBD frequently occurs in MSA but is not mentioned in the diagnostic criteria.

\section{REM sleep behaviour disorder: Clinical presentation, diagnosis}

RBD is a parasomnia disorder manifesting with a loss of physiological atonia in REM sleep and dream enactment. To diagnose RBD, following the International Classification of Sleep Disorders ( $3^{\text {rd }}$ edition), there must be vocalizations and/or complex motor behaviours during REM sleep on videopolysomnography or as part of the patient history. In order to identify REM sleep with loss of physiological atonia, polysomnography must be performed (American Academy of Sleep Medicine, 2014).

RBD manifests frequently in PD, LBD, MSA, and in narcolepsy. Rarely, RBD can be caused by focal brain lesions and neuroimmune diseases (Manni et al., 2011). In such cases, where a disease mentioned above is present, RBD is referred to as secondary. In the absence of a demonstrable disorder causing RBD, this disorder is considered as idiopathic (iRBD) (Ferini-Strambi et al., 2014a).

\section{Idiopathic REM sleep disorder: Phenoconversion to clinically manifest synucleinopathy syndromes}

In 1996, the first iRBD prospective study investigated 29 patients in Minnesota. Initially, the phenoconversion to clinically manifest synucleinopathy syndromes was found to be $38 \%$ following a mean interval from RBD symptoms to neurodegeneration of 3.7 years (Schenck et al., 1986). Several years later in 2013, further follow up showed an even higher $81 \%$ conversion rate after up to 29 years, with a mean conversion time of 14 years after iRBD onset (Schenck et al., 2013).

Another prospective cohort of iRBD encompassing 174 patients was followed between 1991 and 2013 in Spain. The median age at diagnosis of iRBD was 69 years, with a median conversion time of 11 years to a manifest neurodegenerative disorder (Iranzo et al., 2014). Interestingly, like in previous studies, there was a large variation in the conversion interval from iRBD to a defined synucleinopathy syndrome; in some cases, it took as little as 2 years, while in others it took up to 24 years from symptoms onset. This variation encouraged a closer analysis of the differing phenotypes of individual iRBD patients in attempt to assess the probability of early phenoconversion. To compare the integrity of the nigrostriatal pathway in iRBD patients and controls using DAT imaging techniques, the uptake of loflupane was measured in a 3-year time frame. Patients with iRBD had lower loflupane uptake compared to controls, moreover only patients in the iRBD group converted to PD (Iranzo et al., 2011).

In Canada, a further 10-year prospective study of 89 patients with iRBD was published in 2015. In this cohort, the total conversion to a neurodegenerative synucleinopathy was $66 \%$ after 7.5 years. In this study, several symptoms including 
olfactory dysfunction, cognition, depression, anxiety and sleep problems were analysed as markers of phenoconversion. From this analysis, it emerged that hyposmia, a higher age, colour vision dysfunction and motor abnormalities increase the risk of early phenoconversion to synucleinopathy in patients with iRBD (Postuma et al., 2015b). Patients taking anti-depressants with iRBD displayed a lower rate of synucleinopathy conversion, nevertheless they still displayed neurodegenerative symptoms, indicating that antidepressants may unveil iRBD at an earlier stage of the same neurodegenerative process (Postuma and Montplaisir, 2014).

In China, a prospective study of 43 patients with iRBD showed a phenoconversion of $42 \%$ to a synucleinopathy after a median interval of 10.5 years from symptom onset. Patients with higher scores on the Nonmotor symptoms questionnaire (NMSQ), higher scores on Scale for Outcomes in Parkinson DiseaseAutonomic (SCOPA-AUT) and a lower uptake of the DAT binding substrate in the left striatum had a significantly shorter neurodegeneration free survival time. Among specific non-motor symptoms, constipation and olfactory dysfunction increased the risk of phenoconversion, but the results were not statistically significant, possibly because of the small sample size (Li et al., 2017).

So far, there has been a lack of studies evaluating the phenoconversion of iRBD to synucleinopathies in the central and eastern European Slavic population. According to our own data, median age of iRBD symptoms onset was 62 years in a retrospective cohort of 34 Czech subjects with iRBD. In this cohort, the median conversion time to parkinsonism or dementia was 5 years (ranging from 1 to 27 years) from the diagnosis (Peřinová et al., 2018). Further studies are required to evaluate the Czech iRBD population with greater accuracy.

Taking into account the above discussed studies, iRBD has been proven to represent a considerable risk for developing a neurodegenerative disorder. Once iRBD is diagnosed, there are no validated scales to monitor iRBD progression. Thus, the intensity of iRBD symptoms is not easily quantifiable, moreover, the severity apparently fluctuates over years. However, drawing from recent iRBD studies, there are several biomarkers, which indicate increased likelihood of conversion to neurodegeneration. These signs include: abnormal DAT imaging, autonomic symptoms, mild motor symptoms including speech abnormalities, hyposmia and colour vision dysfunction (Iranzo et al., 2011; Postuma et al., 2011; Ferini-Strambi et al., 2014b; Rusz et al., 2016). The following part of this article, covers current knowledge and unresolved questions on olfaction and colour vision as markers of neurodegeneration in synucleinopathies.

\section{Olfaction: Clinical correlates, methods of investigation, progression of hyposmia in synucleiopathies \\ Olfaction is the ability to process chemosensory information. The olfactory pathway consists of the olfactory epithelium, nerve and bulb. This pathway}


contributes information to the thalamus, hippocampus, orbitofrontal cortex, amygdala and, hypothalamus; structures indispensable for numerous functions such as homeostasis and sleep regulation (Purves, 2004).

Hyposmia is defined as a reduced olfactory function, anosmia represents total loss of olfactory function. The cause of olfactory dysfunction can be divided into obstructive affecting nasal and paranasal sinuses, sensory olfactory loss involving impairment of nerve endings or receptors, and neural olfactory loss attributed to dysfunction of structures from the bulb to primary olfactory cortex. Therefore, an abundant number of causes may result in olfactory dysfunction including allergic disorders of nasal and paranasal sinuses, head traumatic injury, medications, radiotherapy, surgical procedures, psychiatric diseases, intracranial tumours, metabolic disorders, endocrine disorders, infections, intracranial vascular disorders, and neurodegenerative disorders (Doty, 2005).

Along with increasing age, there are corresponding olfactory changes resulting in reduced capacity of odour identification and discrimination. It is therefore essential in clinical studies to compare patients to age equivalent healthy controls.

The most widely used commercially available tests to objectively examine olfaction include, the University of Pennsylvania smell identification test (UPSIT) and the Sniffin' test. The UPSIT entails 40 multiple choice questions, where the patient is asked to identify the encapsulated smell released by scratching the surface with a pen (Doty et al., 1984). The Sniffin test has two versions one with 16 and another with 12 multiple-choice questions. Although it has a lower reliability, it is less time consuming in a clinical setting. There are various other tests available to evaluate olfaction and conversion tables have been produced to compare attained olfactory data (Lawton et al., 2016).

Hyposmia in PD has a high prevalence, affecting 90\% of patients (Doty, 2012). Through the olfactory bulb, the first cranial nerve provides a direct pathway from the periphery to cholinergic and adrenergic systems implicated in the pathophysiology of neurodegenerative disorders. Despite the high sensitivity of hyposmia for alpha-synucleinopathies, it can occur in non-neurologic conditions limiting its specificity for diagnosis of prodromal neurodegeneration. As a result, olfaction abnormalities necessitate combination with other more specific biomarkers such as RBD. In a 64 iRBD patient cohort, the 5-year Kaplan-Meier disease free survival in patients with normal olfaction was $86 \%$ but only $35 \%$ in those with hyposmia (Postuma et al., 2011). This is significant, because it aids in the early identification of patients that are at a higher risk of conversion to manifest synucleinopathies. In a further prospective study of patients with newly diagnosed PD, $46 \%$ of those with hyposmia developed dementia, compared to patients without initial hyposmia in whom dementia was only diagnosed in $21 \%$ (Domellof et al., 2017). This indicates that hyposmia may be a symptom of a more severe or distinct PD phenotype. Patients with severe olfactory dysfunction, compared to those with a less severe olfactory dysfunction, were in the group of patients 
that showed the greatest cognitive decline in a further study. Neuroimaging data supported these findings; in the group of patients with the most severe hyposmia there was marked metabolic reduction in the posterior cingulate, precuneus, medial occipital and parieto-occipital-temporal cortex (Baba et al., 2012). In contrast, normosmic patients with PD have a varying phenotype, with less severe motor deficits and a younger age of onset than patients with olfactory dysfunctions (Lee et al., 2015).

Limited data on olfactory function is available in patients with LBD. Studies evaluating this cohort of patients have found significant olfactory dysfunction. Lewy bodies, in LBD, are widespread throughout the brain including the limbic and cortical areas. This is accompanied with an olfactory deficit similar to that in PD suggesting that the olfactory pathway is severely affected as part of the pathophysiological disease process. In MSA, a marginally lower olfactory function compared to controls has been described. Despite this, in MSA the extent of the olfactory dysfunction does not appear to exceed moderate impairment. This could indicate that the pathological progression in MSA does not differ in propagation, but affects some brain regions to a greater extent, prevailing for example in the brainstem and the cerebellum (Doty, 2012).

According to a recent study, longitudinal follow-up of olfaction in patients with progressive neurodegeneration did not show worsening of olfactory function (Iranzo et al., 2013). This counterintuitive result suggests that despite identifying patients at a higher risk of conversion, olfactory function is not a useful marker for monitoring disease progression. Supplementary studies with longer follow-up are required to assess olfactory dysfunction progression in iRBD and in synucleinopathies to confirm whether olfactory loss reaches a plateau long before neurodegeneration affects other neural structures.

\section{Colour vision: Clinical correlates, method of investigation, progression of colour vision in synucleiopathies}

Colour vision is the ability to identify the four basic colours known as pure hues: red, yellow, green, and blue as well as the colour variations obtainable from them. Colour vision is made possible by the trichromatic system of cone cells. In this system, differing photopigments in each subtype of cone cells detect colours of different wavelengths, namely blue, green and red light sensitive cones (Purves, 2004). Dysfunctions in colour detection and processing can occur at any stage from the ocular apparatus to the visual cortex, rendering a vast number of possible pathologies, divided into acquired and congenital disorders.

Congenital colour vision abnormalities affecting cone cells are classified according to the type of deficient cells. Most congenital deficiencies are inherited in an $X$-linked recessive fashion and, therefore, are more common in men, affecting $8 \%$ of males and $0.5 \%$ females in studied populations. There is usually little lifetime variation and both eyes are affected equally. On the other hand, acquired colour 
vision abnormalities may progress or regress and often do not only affect cone cells. Acquired colour vision abnormalities occur in glaucoma, diabetic neuropathy, optic neuritis and PD among some causes (Simunovic, 2016).

Colour vision tests can be split into: pseudoisochromatic plate tests and matching tests, both useful in the diagnosis of congenital colour blindness, arrangement tests for grading colour deficiencies and naming tests which are mostly used in an occupational setting. To assess acquired colour vision deficiencies, an arrangement test, the Farnsworth-Munsell 100-hue test (FM100) is widely used, as it is able to detect subtle differences in colour vision abilities. In this test, there are 4 sets of caps and in each set the patient attempts to order the caps according to hue (Dain, 2004).

In PD loss of dopaminergic cells in the substantia nigra represents the neuropathological hallmark of the disease, but, notably, other parts of the central nervous system (CNS) including the retina and visual cortex undergo neurodegenerative changes (Armstrong, 2015; Normando et al., 2016). Colour discrimination has been shown to be impaired in PD patients (Muller et al., 1998). Despite this, it remains unclear how retinal function is related to substantia nigra dopamine deficiency and whether and to what extent are retinal dopaminergic pathways affected in PD (Willis and Freelance, 2017). In a study employing optical coherence tomography, it has been shown that retinal thickness is not altered in PD patients with or without visual hallucinations compared to healthy controls (Kopal et al., 2015). In RBD, colour vision abnormalities appear at least 5 years before the onset of parkinsonism and/or dementia. When comparing patients with colour vision abnormalities and without colour vision abnormalities in an iRBD cohort, the Kaplan-Meier 5-year disease free survival was $70 \%$ in those with normal and $26 \%$ in those with abnormal colour vision (Postuma et al., 2011). Chromatic discrimination using the FM100 worsened over time in a PD patient cohort, showing a progressive worsening in line with ongoing neurodegeneration (Diederich et al., 2002). Interestingly, an early PD patient cohort did not show consistent changes in colour discrimination compared to controls, thereby indicating that colour vision abnormalities may represent a specific PD phenotype (Vesela et al., 2001).

A study of PD patients investigated the relationship between cognitive impairment, colour discrimination dysfunction, and anatomical magnetic resonance imaging. A strong link was found between cognitive impairment and alterations of posterior white matter that belongs to the visual processing pathway. Specifically, the white matter pathologies were located in the superior longitudinal fasciculus, right fronto-occipital fasciculus, the splenium, the inferior longitudinal fasciculus and the posterior body of the corpus callosum (Bertrand et al., 2012). These findings provide further insight into the mechanism of colour vision dysfunction, which does not appear to be limited to the visual apparatus. Colour discrimination dysfunction thus signals a widespread disease process, affecting posterior white 
matter.Visual hallucinations in LBD could be explained by the widespread presence of Lewy bodies, including in these posterior white matter locations.

By summarizing these findings, some studies show that, not only do colour vision changes appear a considerable time before the onset of clinically apparent motor or cognitive changes, but also indicate a worse disease prognosis. The examination of the progressive relationship between colour vision, visual hallucinations and cognitive decline, as well as disease progression, in PD and other synucleinopathies, should be a focus of further studies.

\section{Conclusion}

A wide array of biomarkers, have been proven to precede the clinical manifestation of PD and other synucleinopathies. iRBD is the strongest predictor of future clinically manifest neurodegenerative disorder and using other biomarkers, we can further stratify iRBD patients with respect to the risk of early phenoconversion. Clinical markers that increase the likelihood of progression from iRBD to synucleinopathies include decline of tracer uptake on DAT imaging methods as well as motor and non-motor symptoms. Despite the high diagnostic strength of DAT imaging and subtle motor abnormalities, they are not the earliest biomarkers. Non-motor signs of synucleinopathy such as colour vision and olfaction impairment are detectable by clinical examination several years before disease onset.

The timely selection of patients that are at the highest risk of future neurodegeneration allows us to identify and monitor patients that would benefit the most from neuroprotective drugs once they become available. In conclusion, olfaction and colour vision testing can help us select patients with iRBD that are at greatest risk of phenoconversion to PD and other synucleinopathies. An advantage of monitoring these non-motor symptoms is their much earlier time onset compared to other markers of neurodegeneration. Detailed analysis of olfactory and colour vision dysfunction can provide valuable information regarding brain pathologies, further specifying clinical phenotypes, and giving clues to underlying pathophysiological mechanisms in PD and related disorders.

\section{References}

American Academy of Sleep Medicine (2014) International Classification of Sleep Disorders. Armstrong, R. A. (2015) Oculo-visual dysfunction in Parkinson's disease. J. Parkinsons Dis. 5, 715-726.

Baba, T., Kikuchi, A., Hirayama, K., Nishio, Y., Hosokai, Y., Kanno, S., Hasegawa, T., Sugeno, N., Konno, M., Suzuki, K., Takahashi, S., Fukuda, H., Aoki, M., Itoyama, Y., Mori, E., Takeda, A. (2012) Severe olfactory dysfunction is a prodromal symptom of dementia associated with Parkinson's disease: a 3 year longitudinal study. Brain 135, 161-169.

Berg, D., Postuma, R. B., Adler, C. H., Bloem, B. R., Chan, P., Dubois, B., Gasser, T., Goetz, C. G., Halliday, G., Joseph, L., Lang, A. E., Liepelt-Scarfone, I., Litvan, I., Marek, K., Obeso, J., Oertel,W., Olanow, C.W., Poewe, W., Stern, M., Deuschl, G. (2015) MDS research criteria for prodromal Parkinson's disease. Mov. Disord. 30, 1600-1611.

Bernis, M. E., Babila, J. T., Breid, S., Wusten, K. A., Wullner, U., Tamguney, G. (2015) Prion-like propagation of 
human brain-derived alpha-synuclein in transgenic mice expressing human wild-type alpha-synuclein. Acta Neuropathol. Commun. 3, 75.

Bertrand, J. A., Bedetti, C., Postuma, R. B., Monchi, O., Genier Marchand, D., Jubault, T., Gagnon, J. F. (2012) Color discrimination deficits in Parkinson's disease are related to cognitive impairment and white-matter alterations. Mov. Disord. 27, 1781-1788.

Braak, H., Del Tredici, K., Rub, U., De Vos, R. A., Jansen Steur, E. N., Braak, E. (2003) Staging of brain pathology related to sporadic Parkinson's disease. Neurobiol. Aging 24,197-211.

Brooks, D. J., Seppi, K.; Neuroimaging Working Group on M. S. A. (2009) Proposed neuroimaging criteria for the diagnosis of multiple system atrophy. Mov. Disord. 24, 949-964.

Dain, S. J. (2004) Clinical colour vision tests. Clin. Exp. Optom. 87, 276-293.

Diederich, N. J., Raman, R., Leurgans, S., Goetz, C. G. (2002) Progressive worsening of spatial and chromatic processing deficits in Parkinson disease. Arch. Neurol. 59, 1249-1252.

Domellof, M. E., Lundin, K. F., Edstrom, M., Forsgren, L. (2017) Olfactory dysfunction and dementia in newly diagnosed patients with Parkinson's disease. Parkinsonism Relat. Disord. 38, 41-47.

Doty, R. L. (2005) Clinical studies of olfaction. Chem. Senses 30, i207-i209 (Suppl. 1).

Doty, R. L. (2012) Olfaction in Parkinson's disease and related disorders. Neurobiol. Dis. 46, 527-552.

Doty, R. L., Shaman, P., Kimmelman, C. P., Dann, M. S. (1984) University of Pennsylvania Smell Identification Test: a rapid quantitative olfactory function test for the clinic. Laryngoscope 94, 176-178.

Ferini-Strambi, L., Marelli, S., Galbiati, A., Rinaldi, F., Giora, E. (2014a) REM Sleep Behavior Disorder (RBD) as a marker of neurodegenerative disorders. Arch. Ital. Biol. 152, 129-146.

Ferini-Strambi, L., Oertel, W., Dauvilliers, Y., Postuma, R. B., Marelli, S., Iranzo, A., Arnulf, I., Hogl, B., Manni, R., Miyamoto, T., Fantini, M. L., Puligheddu, M., Jennum, P., Sonka, K., Santamaria, J., Zucconi, M., Rancoita, P. M., Leu-Semenescu, S., Frauscher, B., Terzaghi, M., Miyamoto, M., Unger, M., Stiasny-Kolster, K., Desautels, A., Wolfson, C., Pelletier, A., Montplaisir, J. (2014b) Autonomic symptoms in idiopathic REM behavior disorder: a multicentre case-control study.J. Neurol. 261, 1112-1118.

Gilman, S., Wenning, G. K., Low, P. A., Brooks, D. J., Mathias, C. J., Trojanowski, J. Q., Wood, N.W., Colosimo, C., Durr, A., Fowler, C. J., Kaufmann, H., Klockgether, T., Lees, A., Poewe, W., Quinn, N., Revesz, T., Robertson, D., Sandroni, P., Seppi, K., Vidailhet, M. (2008) Second consensus statement on the diagnosis of multiple system atrophy. Neurology 71, 670-676.

Goedert, M., Spillantini, M. G., Del Tredici, K., Braak, H. (2013) 100 years of Lewy pathology. Nat. Rev. Neurol. 9, 13-24.

Halliday, G. M., Holton, J. L., Revesz, T., Dickson, D.W. (2011) Neuropathology underlying clinical variability in patients with synucleinopathies. Acta Neuropathol. 122, 187-204.

Iranzo, A., Valldeoriola, F., Lomena, F., Molinuevo, J. L., Serradell, M., Salamero, M., Cot, A., Ros, D., Pavia, J., Santamaria, J., Tolosa, E. (2011) Serial dopamine transporter imaging of nigrostriatal function in patients with idiopathic rapid-eye-movement sleep behaviour disorder: a prospective study. Lancet Neurol. 10, 797-805.

Iranzo, A., Serradell, M., Vilaseca, I., Valldeoriola, F., Salamero, M., Molina, C., Santamaria, J., Tolosa, E. (2013) Longitudinal assessment of olfactory function in idiopathic REM sleep behavior disorder. Parkinsonism Relat. Disord. 19, 600-604.

Iranzo, A., Fernandez-Arcos, A., Tolosa, E., Serradell, M., Molinuevo, J. L., Valldeoriola, F., Gelpi, E., Vilaseca, I., Sanchez-Valle, R., Llado, A., Gaig, C., Santamaria, J. (2014) Neurodegenerative disorder risk in idiopathic REM sleep behavior disorder: study in 174 patients. PLoS One 9, e89741.

Kopal, A., Mejzlikova, E., Preiningerova, J. L., Brebera, D., Ulmanova, O., Ehler, E., Roth, J. (2015) Changes of retina are not involved in the genesis of visual hallucinations in Parkinson's disease. Parkinsons Dis. 2015 709191. 
Lawton, M., Hu, M. T., Baig, F., Ruffmann, C., Barron, E., Swallow, D. M., Malek, N., Grosset, K. A., Bajaj, N., Barker, R. A., Williams, N., Burn, D. J., Foltynie, T., Morris, H. R., Wood, N.W., May, M. T., Grosset, D. G., Ben-Shlomo, Y. (2016) Equating scores of the University of Pennsylvania Smell Identification Test and Sniffin' Sticks test in patients with Parkinson's disease. Parkinsonism Relat. Disord. 33, 96-101.

Lee, D. H., Oh, J. S., Ham, J. H., Lee, J. J., Lee, I., Lee, P. H., Kim, J. S., Sohn, Y. H. (2015) Is normosmic Parkinson disease a unique clinical phenotype? Neurology 85, 1270-1275.

Li, Y., Kang, W., Yang, Q., Zhang, L., Zhang, L., Dong, F., Chen, S., Liu, J. (2017) Predictive markers for early conversion of iRBD to neurodegenerative synucleinopathy diseases. Neurology 88, 1493-1500.

Mahlknecht, P., Seppi, K., Poewe, W. (2015) The concept of prodromal Parkinson's disease. J. Parkinsons Dis. 5, 681-697.

Manni, R., Ratti, P. L., Terzaghi, M. (2011) Secondary “incidental” REM sleep behavior disorder: do we ever think of it? Sleep Med. 12, S50-S53 (Suppl. 2).

Marques, O., Outeiro, T. F. (2012) Alpha-synuclein: from secretion to dysfunction and death. Cell Death Dis. 3, e350.

McKeith, I. G., Boeve, B. F., Dickson, D.W., Halliday, G., Taylor, J. P., Weintraub, D., Aarsland, D., Galvin, J., Attems, J., Ballard, C. G., Bayston, A., Beach, T. G., Blanc, F., Bohnen, N., Bonanni, L., Bras, J., Brundin, P., Burn, D., Chen-Plotkin, A., Duda, J. E., El-Agnaf, O., Feldman, H., Ferman, T. J., Ffytche, D., Fujishiro, H., Galasko, D., Goldman, J. G., Gomperts, S. N., Graff-Radford, N. R., Honig, L. S., Iranzo, A., Kantarci, K., Kaufer, D., Kukull, W., Lee, V. M.Y., Leverenz, J. B., Lewis, S., Lippa, C., Lunde, A., Masellis, M., Masliah, E., Mclean, P., Mollenhauer, B., Montine, T. J., Moreno, E., Mori, E., Murray, M., O’Brien, J. T., Orimo, S., Postuma, R. B., Ramaswamy, S., Ross, O. A., Salmon, D. P., Singleton, A., Taylor, A., Thomas, A., Tiraboschi, P., Toledo, J. B., Trojanowski, J. Q., Tsuang, D., Walker, Z., Yamada, M., Kosaka, K. (2017) Diagnosis and management of dementia with Lewy bodies: Fourth consensus report of the DLB Consortium. Neurology 89, 88-100.

Muller, T., Kuhn, W., Buttner, T., Eising, E., Coenen, H., Haas, M., Przuntek, H. (1998) Colour vision abnormalities do not correlate with dopaminergic nigrostriatal degeneration in Parkinson's disease. J. Neurol. 245, 659-664.

Normando, E. M., Davis, B. M., De Groef, L., Nizari, S., Turner, L. A., Ravindran, N., Pahlitzsch, M., Brenton, J., Malaguarnera, G., Guo, L., Somavarapu, S., Cordeiro, M. F. (2016) The retina as an early biomarker of neurodegeneration in a rotenone-induced model of Parkinson's disease: Evidence for a neuroprotective effect of rosiglitazone in the eye and brain. Acta Neuropathol. Commun. 4, 86.

Peřinová, P., Plchová, P., Bušková, J., Kemlink, D., Ibarburu Lorenzo Y. Losada, V., Dostálová, S., Vorlová, T., Šonka, K. (2018) Patients with idiopathic REM sleep behavior disorder follow-up - Phenoconversion into parkinsonian syndrome and dementia. Cesk. Slov. Neurol. N 2, 205-207.

Postuma, R. B., Montplaisir, J.Y. (2014) Antidepressants and REM behavior disorder. Sleep 37, 1395.

Postuma, R. B., Berg, D. (2016) Advances in markers of prodromal Parkinson disease. Nat. Rev. Neurol. 12, 622-634.

Postuma, R. B., Gagnon, J. F., Vendette, M., Desjardins, C., Montplaisir, J.Y. (2011) Olfaction and color vision identify impending neurodegeneration in rapid eye movement sleep behavior disorder. Ann. Neurol. 69 , 811-818.

Postuma, R. B., Berg, D., Stern, M., Poewe, W., Olanow, C.W., Oertel,W., Obeso, J., Marek, K., Litvan, I., Lang, A. E., Halliday, G., Goetz, C. G., Gasser, T., Dubois, B., Chan, P., Bloem, B. R., Adler, C. H., Deuschl, G. (2015a) MDS clinical diagnostic criteria for Parkinson's disease. Mov. Disord. 30, 1591-1601.

Postuma, R. B., Gagnon, J. F., Bertrand, J. A., Genier Marchand, D., Montplaisir, J.Y. (2015b) Parkinson risk in idiopathic REM sleep behavior disorder: preparing for neuroprotective trials. Neurology 84, 1104-1113.

Purves, D. (2004) Neuroscience. Sinauer Associates, Sunderland. 
96) Prague Medical Report / Vol. 119 (2018) No. 2-3, p. 85-96

Rusz, J., Hlavnicka, J., Tykalova, T., Buskova, J., Ulmanova, O., Ruzicka, E., Sonka, K. (2016) Quantitative assessment of motor speech abnormalities in idiopathic rapid eye movement sleep behaviour disorder. Sleep Med. 19, 141-147.

Schenck, C. H., Bundlie, S. R., Ettinger, M. G., Mahowald, M. W. (1986) Chronic behavioral disorders of human REM sleep: a new category of parasomnia. Sleep 9, 293-308.

Schenck, C. H., Boeve, B. F., Mahowald, M.W. (2013) Delayed emergence of a parkinsonian disorder or dementia in $81 \%$ of older men initially diagnosed with idiopathic rapid eye movement sleep behavior disorder: a 16-year update on a previously reported series. Sleep Med. 14, 744-748.

Simunovic, M. P. (2016) Acquired color vision deficiency. Surv. Ophthalmol. 61, 132-155.

Spillantini, M. G., Goedert, M. (2000) The alpha-synucleinopathies: Parkinson's disease, dementia with Lewy bodies, and multiple system atrophy. Ann. N.Y. Acad. Sci. 920, 16-27.

Uchihara, T., Giasson, B. I. (2016) Propagation of alpha-synuclein pathology: Hypotheses, discoveries, and yet unresolved questions from experimental and human brain studies. Acta Neuropathol. 131, 49-73.

Vesela, O., Ruzicka, E., Jech, R., Roth, J., Stepankova, K., Mecir, P., Solano, Z., Preclikova, E. (2001) Colour discrimination impairment is not a reliable early marker of Parkinson's disease. J. Neurol. 248, 975-978.

Willis, G. L., Freelance, C. B. (2017) Neurochemical systems of the retina involved in the control of movement. Front. Neurol. 8, 324. 